TITLE:

\title{
Dynamic expression and roles of Hes factors in neural development.
}

$\operatorname{AUTHOR}(S)$ :

Kageyama, Ryoichiro; Shimojo, Hiromi; Imayoshi, Itaru

\section{CITATION:}

Kageyama, Ryoichiro ...[et al]. Dynamic expression and roles of Hes factors in neural development.. Cell and tissue research 2014, 359(1): 125-133

\section{ISSUE DATE:}

2014-05-22

URL:

http://hdl.handle.net/2433/198461

\section{RIGHT:}

The final publication is available at Springer via http://dx.doi.org/10.1007/s00441-0141888-7.; この論文は出版社版でありません。引用の際には出版社版をご確認ご利用くだ さい。; This is not the published version. Please cite only the published version. 


\section{Dynamic expression and roles of Hes factors in neural development}

Ryoichiro Kageyama ${ }^{1,2,3 *}$, Hiromi Shimojo ${ }^{1,2,3}$, and Itaru Imayoshi ${ }^{1,3,4}$

${ }^{1}$ Institute for Virus Research, Kyoto University, Shogoin-Kawahara, Sakyo-ku, Kyoto 606-8507, Japan; ${ }^{2}$ Japan Science and Technology Agency, CREST, Shogoin-Kawahara, Sakyo-ku, Kyoto 606-8507, Japan; ${ }^{3}$ World Premier International Research Initiative-Institute for Integrated Cell-Material Sciences (WPI-iCeMS), Kyoto University, Kyoto 606-8501, Japan; ${ }^{4}$ The Hakubi Center, Kyoto University, Kyoto 606-8302, Japan

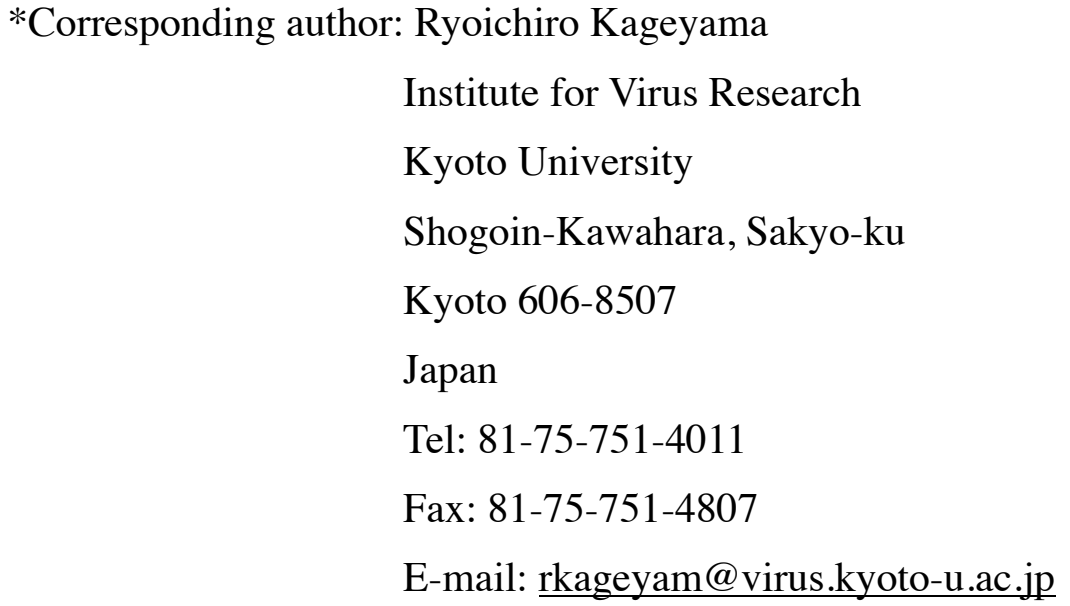

Running title: Dynamic bHLH factors

Keywords: basic helix-loop-helix factor, Hes1, neural progenitor cell, Notch signaling, oscillatory expression, proneural factor 


\section{Summary}

The basic helix-loop-helix factors (bHLH) Hes1 and Hes5 repress the expression of proneural factors such as Ascl1, thereby inhibiting neuronal differentiation and maintaining neural progenitor cells (NPCs). Hes1 expression oscillates by negative feedback with a period of about 2-3 hours in proliferating NPCs. Induction of sustained expression of Hes1 in NPCs inhibits their cell cycle progression, suggesting that the oscillatory expression of Hes1 is important for the proliferation of NPCs. Hes1 oscillation drives the oscillatory expression of proneural factors such as Ascl1 by periodic repression. By contrast, in differentiating neurons, Hes 1 expression disappears and the expression of proneural factors is up-regulated and sustained. A new optogenetics approach that induces Ascl1 expression by blue light illumination demonstrated that sustained expression of Ascl1 induces neuronal differentiation, whereas oscillatory expression of Ascl1 activates proliferation of NPCs. These results together indicate that Hes1 regulates the oscillatory versus sustained expression of the proneural factor Ascl1, which regulates the proliferation of NPCs and the subsequent processes of cell cycle exit and neuronal fate determination, depending on the expression dynamics.

\section{Introduction}

Neuroepithelial cells, the first form of embryonic neural progenitor cells (NPCs), extend from the ventricular surface to the pial surface of the neural tube. These cells proliferate extensively by repeating symmetric cell division, where each neuroepithelial cell divides into two neuroepithelial cells (Fig. 1) (Alvarez-Buylla et al. 2001; Fishell and Kriegstein 2003; Fujita 2003; Götz and Huttner 2005; Miller and Gauthier 2007; Kriegstein and Alvarez-Buylla, 2009). As the wall of the neural tube becomes thicker, neuroepithelial cells are gradually elongated, becoming radial glial cells, which have cell bodies in the innermost region, called the ventricular zone, and radial fibers reaching the pial surface (Fig. 1). Radial glial cells undergo asymmetric cell division, where each radial glial cell divides into two distinct cell types, a new radial glial cell and an immature neuron or a basal progenitor (Fig. 1) (Malatesta et al. 2000; Miyata et al. 2001; Noctor et al. 2001). Immature neurons migrate outside of the ventricular zone along radial fibers into the cortical plate, where these cells become mature neurons. On the other hand, basal progenitors migrate into the subventricular zone, proliferate further, 
and produce more neurons. Radial glial cells sequentially give rise to different types of neurons, initially deep layer neurons and then superficial layer neurons, by repeating asymmetric cell divisions (Fig. 1). Later, radial glial cells give rise to oligodendrocytes, ependymal cells, and astrocytes (Fig. 1). Both neuroepithelial and radial glial cells are considered embryonic NPCs.

It has been shown that basic helix-loop-helix (bHLH) factors play key roles in maintenance of NPCs and their cell fate determination (Bertrand et al., 2002; Ross et al., 2003; Wilkinson et al., 2013). In this review, we will discuss the recent findings regarding the complex regulations and functions of bHLH factors in neural development.

\section{Maintenance of NPCs}

The bHLH factors Hes1 and Hes5, mammalian homologues of Drosophila hairy and Enhancer of split (E(spl)), are highly expressed by NPCs. These factors form homo- or heterodimers through the helix-loop-helix domain and bind to the DNA sequences, such as the $\mathrm{N}$ box (CACNAG) and the class $\mathrm{C}$ site $(\mathrm{CACG}(\mathrm{C} / \mathrm{A}) \mathrm{G})$, through the basic region (Sasai et al., 1992). These factors act as transcriptional repressors by recruiting the co-repressor Transducin-like E(spl) (TLE1-4)/Groucho-related gene (Grg), a homologue of Drosophila Groucho, at the carboxy-terminal WRPW domain (Trp-Arg-Pro-Trp) (Akazawa et al. 1992; Sasai et al. 1992; Grbavec and Stifani, 1996). The best-studied target genes for Hes1 and Hes5 are proneural genes such as Ascl1/Mash1 and Neurogenin2 (Neurog2), which promote neuronal fate determination (Bertrand et al., 2002; Ross et al., 2003; Wilkinson et al., 2013). Hes1 and Hes5 directly repress the proneural gene expression, thereby inhibiting neuronal differentiation and leading to the maintenance of NPCs. In the absence of either Hes 1 or Hes5, the defects are minimum, but in the absence of both Hesl and Hes5, proneural gene expression is up-regulated, accelerating neuronal differentiation and leading to premature exhaustion of NPCs (Ishibashi et al., 1995; Ohtsuka et al., 1999; Hatakeyama et al., 2004). However, there are some NPCs remaining even in the absence of Hesl and Hes5 in the dorsal regions of the developing nervous system, where Hes3 is expressed. In the absence of Hes 1, Hes3, and Hes5, virtually all NPCs are depleted in most of the developing nervous system except for the telencephalon (Hatakeyama et al. 2004). Thus, Hes1, Hes3, and Hes5 cooperatively regulate the maintenance of NPCs, but another Hes-related factor may be 
also responsible for the maintenance of NPCs in the telencephalon.

In differentiating neurons, proneural factors induce the expression of Notch ligands such as the transmembrane proteins Delta-like1 (Dll1), which activate Notch receptors such as the transmembrane protein Notch1 in neighboring cells (Castro et al. 2006). Upon activation of Notch signaling, Notch receptors undergo successive cleavages, releasing the Notch intracellular domain (NICD) from the transmembrane domain (Fig. 2). NICD next moves to the nucleus and forms a complex with the DNA-binding protein Rbpj and the transcriptional co-activator Maml (Fig. 2). This ternary complex (NICD-Rbpj-Maml) activates target genes such as Hes1 and Hes5 (Jarriault et al. 1995; Fortini 2009; Honjo 1996; Kageyama et al. 2008; Kopan and Ilagan 2009; Pierfelice et al. 2011). Then, Hes1 and Hes5 inhibit neuronal differentiation and maintain NPCs (Ishibashi et al. 1994; Ohtsuka et al. 1999; Hatakeyama et al. 2004; Kageyama et al. 2007). Thus, differentiating neurons inhibit neighboring cells from differentiating into the same cell types via Notch signaling, a process called lateral inhibition. In the absence of Notch signaling, all cells express proneural genes and initiate neuronal differentiation, resulting in premature depletion of NPCs (Ishibashi et al. 1995; Hatakeyama et al. 2004; Imayoshi et al. 2010). Thus, Notch signaling is essential to maintain NPCs in the developing nervous system.

The Notch signaling pathway suggests that differentiating neurons that express Notch ligands are required in the neighbor to maintain NPCs, raising a question as to how NPCs are maintained during early stages of development before neurons are born. Another issue is that proneural factors like Ascl1 have contradictory functions. As a proneural factor, Ascl1 induces cell cycle exit and promotes neuronal differentiation. However, Ascl1 is expressed by NPCs, and its absence results in slow proliferation of these cells, suggesting that Ascl1 is involved in proliferation of NPCs. Indeed, it was found that Ascl1 directly activates the expression of genes involved in cell cycle progression, raising a question as to how Ascl1 coordinates such contradictory function, activation of NPC proliferation and induction of cell cycle exit and neuronal differentiation. It was also found that Notch ligand genes like Dll1 are expressed in a salt-and-pepper pattern by NPCs, suggesting that Notch signaling is active before neurons are born. We recently found that the expression of these factors is more dynamic than previously thought, and that the dynamics of gene expression is important for the functions of these factors. 


\section{Oscillatory expression of Notch signaling genes}

We previously found that Hes 1 expression oscillates with a period of about 2-3 hours in many cell types such as fibroblasts after serum stimulation or Notch activation (Hirata et al. 2002). This oscillatory expression is soon dampened after three or four cycles. However, time-lapse imaging analyses using a Hes1 reporter revealed that Hes1 oscillation is dampened at the population level but not at a single cell level; Hes1 oscillation continues in individual cells but soon becomes out of synchrony (Masamizu et al., 2006). Hes1 oscillation is regulated by negative feedback with a delayed timing (Fig. 3) (Hirata et al. 2002). Hes1 represses its own expression by directly binding to multiple $\mathrm{N}$ box sequences (CACNAG) of its promoter (Takebayashi et al. 1994). When the promoter is repressed, hes $1 \mathrm{mRNA}$ and Hes1 protein disappear rapidly because they are extremely unstable, and the disappearance of Hes 1 protein allows the next round of its expression. In this way, Hes1 expression autonomously oscillates with a period of about 2-3 hours (Hirata et al. 2002).

Time-lapse imaging analysis revealed that Hesl expression also oscillates in NPCs (Fig. 4) (Shimojo et al. 2008). Hes1 expression exhibits an inverse correlation with Ascl1 and Neurog2 protein and Dll1 mRNA expression in NPCs (Baek et al., 2006; Shimojo et al. 2008), suggesting that Hes1 oscillation induces the oscillatory expression of Ascl1, Neurog2, and Dll1 by periodic repression. Time-lapse imaging analysis revealed that Ascl1, Neurog2 and Dll1 expression indeed oscillates in NPCs, where Hes1 expression oscillates (Fig. 4) (Shimojo et al. 2008; Imayoshi et al., 2013). However, in differentiating neurons, where Hes1 expression disappears, Ascl1, Neurog2 and Dll1 expression becomes sustained (Fig. 4) (Shimojo et al. 2008; Imayoshi et al., 2013). It is likely that the proneural factors Ascl1 and Neurog2 cannot induce neuronal differentiation when their expression oscillates, probably because many of their downstream genes do not respond to Ascl1 and Neurog2 oscillation, and that Ascl1 and Neurog2 induce neuronal differentiation only when their expression becomes sustained. When Ascl1 and Neurog2 expression oscillates, only rapidly responding genes such as Dll1 may be selectively induced, and Dll1 oscillations may lead to the mutual activation of Notch signaling and the maintenance of NPCs. Agreeing with this notion, Neurog 2 is phosphorylated by cyclin-dependent kinases in NPCs, and phosphorylated Neurog 2 can induce Dll1 expression efficiently but not other gene expression (Ali et al. 2011; 
Hindley et al. 2012). Only non-phosphorylated Neurog2 can induce the expression of neuronal differentiation genes. These results suggest that Neurog 2 may lead to two opposite outcomes, depending on its expression dynamics and phosphorylation status: when it is phosphorylated and its expression oscillates, Neurog2 induces the maintenance of NPCs, but when it is dephosphorylated and its expression is sustained, Neurog2 induces neuronal differentiation.

Oscillatory versus sustained expression of proneural genes may involve the stability of gene products. For example, it was previously shown that the oscillatory expression of a Hesl-related gene, Hes7, turns into sustained expression, when its product is stabilized by introduction of a point mutation, indicating that the instability of gene products is required for oscillatory expression (Hirata et al. 2004). In NPCs, Neurog2 mRNA is destabilized by the RNase III Drosha, and loss of Drosha stabilizes Neurog2 mRNA, thereby promoting neuronal differentiation (Knuckles et al., 2012). Similarly, Ascl1 protein is destabilized by NICD in NPCs, but it is stabilized in neurons, where NICD is absent (Sriuranpong et al., 2002). These results suggest that the stability of proneural gene products is differentially controlled between NPCs and neurons, thereby enabling different expression dynamics.

The above results suggest that salt-and-pepper patterns of Ascl1, Neurog2, and Dll1 in NPCs are the result of oscillatory expression. It is generally thought that Asc11-, Neurog2-, or Dl11-positive cells are selected to become neurons first while negative cells remain undifferentiated. However, their expression just oscillates, and positive and negative cells may be equivalent to each other (Kageyama et al., 2008). We speculate that Dll1 oscillation enables the maintenance of NPCs by mutual activation of Notch signaling without the aid of neurons. These results suggest that Notch signaling functions not only by a one-way mechanism (neuron to NPC) but also by reciprocal transmission (NPC to NPC).

\section{Optogenetic approach to control gene expression dynamics}

The above results also suggest that the oscillatory versus sustained expression patterns of proneural factors are important for their activities, NPC proliferation versus neuronal differentiation. To examine this notion further, we adopted an optogenetic approach using a hybrid protein, GAVPO. GAVPO is a light-activatable protein consisting of the light-inducible dimerizing protein Vivid (VVD), a Gal4 DNA-binding domain, and a 
p65 transcriptional activation domain (Wang et al., 2012). A dimer form, but not a monomer form, of the Gal4 DNA-binding domain can interact with UAS sequences. Blue light illumination activates VVD, forming a dimer, and a dimer form of the Gal4 DNA-binding domain binds to the UAS sequences. Then, the p65 transcriptional activation domain up-regulates the gene expression under the control of the UAS sequences. The significance of gene expression dynamics was examined by introducing the light-dependent Ascl1-inducible system into Ascl1-null NPCs. Light-induced sustained Ascl1 expression promotes neuronal differentiation, whereas light-induced oscillatory Ascl1 expression with 3-hour periodicity activates proliferation of NPCs. These results clearly showed that depending on the expression dynamics, the proneural factor Ascl1 can exhibit opposite functions: oscillatory Ascl can activate NPC proliferation, whereas sustained Ascl1 can induce neuronal differentiation (Imayoshi et al., 2013).

We also found that the bHLH factors Hes1 and Olig2 are expressed in an oscillatory manner by NPCs, whereas their expression becomes dominant and sustained during astrocyte and oligodendrocyte differentiation, respectively (Imayoshi et al., 2013). These results suggest that multipotency is a state of oscillatory expression of multiple fate-determining bHLH factors. By contrast, the fate choice is a process of sustained expression of a selected single bHLH factor. The mechanisms of how oscillatory bHLH factors specifically activate the expression of genes involved in cell cycle progression and of how one of the fate-determining bHLH factors is selected and up-regulated during cell fate choice remain to be analyzed.

\section{Basal progenitors and outer subventricular zone (OSVZ) progenitors}

It was recently found that in addition to basal progenitors, outer SVZ (OSVZ) progenitors are formed (Hansen et al., 2010; Fietz et al., 2010; Shitamukai et al., 2011). Basal progenitors formed by Tbr2 migrate into the SVZ, retract their apical and basal processes and usually divide only once to generate two neurons (Sessa et al., 2008). In these cells, Hesl and Hes5 expression is down-regulated, suggesting that Notch signaling is not active (Fig. 5) (Mizutani et al., 2007; Kawaguchi et al., 2008). OSVZ progenitors have radial fibers extending to the pial surface but lack apical processes (Fig. 5). In contrast to basal progenitors, OSVZ progenitors repeatedly undergo asymmetric cell division, forming another OSVZ progenitor and a post-mitotic neuron (Hansen et 
al., 2010; Fietz et al., 2010). The latter cell expresses Notch ligands and activates Notch signaling in its sibling OSVZ progenitor (Fig. 5) (Shitamukai et al., 2011). Indeed, OSVZ progenitors express Hes1, and inhibition of Notch signaling by treatment with a $\gamma$-secretase inhibitor induces OSVZ progenitors to differentiate into neurons or $\mathrm{Tbr}^{+}$ basal progenitors (Hansen et al., 2010), suggesting that Notch signaling is required for maintenance of OSVZ progenitors (Fig. 5).

It was shown that the developing human neocortex has an expanded outer region in the SVZ, suggesting that OSVZ progenitors are responsible for the expansion of the cortex. It is possible that the cells that migrate into the SVZ may become OSVZ progenitors when Notch signaling is active, whereas they may become basal progenitors when Notch signaling is inactive. It remains to be determined how Notch signaling is regulated in the SVZ and whether Hes1 expression oscillates in OSVZ progenitors, as observed in radial glial cells.

\section{Sustained Hes1 expression in boundary cells}

The developing nervous system is partitioned into many compartments by boundaries such as the isthmus and the zona limitans intrathalamica (Fig. 6). The nervous system is also partitioned into the right and left halves by the roof plate and the floor plate (Fig. 6). These boundaries function as the signaling centers by expressing signaling molecules such as Fgf8, Shh and Wnt and regulate region-specific specification of NPCs and neurons in neighboring compartments (Kiecker and Lumsden 2005). Cells in these boundary regions usually do not proliferate actively or do not give rise to neurons. Thus, the proliferation and differentiation characteristics are different between boundary cells and compartmental NPCs.

Cells in boundary regions express Hes1 at high levels in a sustained manner (Fig. 6) (Baek et al. 2006). Sustained and high levels of Hes1 expression repress the expression of proneural genes and cell cycle regulators such as cyclin D1 and cyclin E2 (Shimojo et al. 2008). As a result, both NPC proliferation and neuronal differentiation are inhibited by sustained Hes1 expression (Baek et al. 2006). By contrast, when Hes genes are inactivated, cells in the boundary regions ectopically express proneural genes and differentiate into neurons (Hirata et al. 2001; Baek et al. 2006). These results suggest that cells with sustained Hes1 expression are rather dormant with regard to proliferation and differentiation, and that oscillatory expression of Hes1 may be 
important for proliferation and differentiation of NPCs. This feature is similar to the one observed in fibroblasts, where sustained Hes1 overexpression inhibits their proliferation, leading to reversible quiescence (Yoshiura et al., 2007; Sang et al. 2008).

The mechanism by which oscillatory versus sustained Hes1 expression is regulated remains to be determined. In fibroblasts, blockade of Jak-Stat signaling by treatment with a Jak inhibitor dampens Hes1 oscillations by stabilizing the Hes1 protein, resulting in steady Hes1 expression in fibroblasts (Yoshiura et al. 2007). Similarly, treatment with a Jak inhibitor inhibits Hes1 oscillations in NPCs, suggesting that Jak-Stat signaling is involved in the regulation of Hes1 oscillations in these cells (Shimojo et al. 2008).

\section{Downstream events of oscillatory expression}

As described above, Dll1 is expressed in an oscillatory manner via periodic repression by Hes1 and periodic activation by Ascl1 and Neurog2. However, if downstream factors are stable, they cannot respond in an oscillatory manner but may gradually accumulate in a step-wise manner. When the levels of downstream factors reach certain values, new events may happen. In this case, Hes1, Ascl1, and Neurog2 oscillators may function as cellular clocks to regulate the timing of certain events. However, this possibility remains to be further analyzed. Other downstream factors may be gradually down-regulated over time in response to Hes1, Ascl1, and Neurog2 oscillations. It was previously shown that sustained overexpression of Hes1 or Hes5 accelerates astrocyte formation (Ohtsuka et al. 2001), raising the possibility that compared to Hes1 oscillation, sustained Hes 1 expression accelerates the transition from neurogenesis to astrogenesis. Identification of downstream genes for Hes1, Ascl1, and Neurog2 oscillators will be required to understand whether these oscillators are involved in such fate transition.

One candidate gene involved in the transition from neurogenesis to astrogenesis is ESET/Setdb1/KMT1E, a histone H3 Lys-9 (H3K9) methyltransferase gene. This gene has multiple Hes1-binding sites in the promoter, although it remains to be determined whether Hes1 regulates ESET expression. ESET is highly expressed by NPCs at early stages of development, but the expression is down-regulated over time and becomes almost absent at later stages when the transition from neurogenesis to astrogenesis occurs (Tan et al. 2012). Inactivation of ESET enhances astrocyte formation at the expense of neurogenesis. Conversely, overexpression of ESET 
decreases the astrocyte differentiation (Tan et al. 2012). Another regulator for the transition from neurogenesis to astrogenesis is the Polycomb group complex, although it is unknown whether its expression is controlled by Hes1 and proneural factors. Inactivation of this complex prolongs neurogenesis and delays astrogenesis, indicating that the Polycomb group complex regulates the fate switch of NPCs from neurogenesis to astrogenesis (Hirabayashi et al. 2009). These results suggest that decreasing ESET activity and increasing Polycomb activity during development may be under the control of internal clock mechanisms that regulate the timing of cell fate switches from neurogenesis to astrogenesis. Further analyses will be required to determine whether Hes 1 and proneural factors are involved in regulation of ESET and Polycomb factor expression.

\section{Conclusions and Perspectives}

The oscillatory versus sustained expression dynamics of bHLH factors is very important for their activities. When Hes1 expression oscillates, NPCs proliferate actively. However, when the expression is sustained, NPCs do not proliferate well, resulting in a dormant state. Alternatively, at later stages, these cells differentiate into astrocytes. Similarly, Ascl1 exhibits contradictory functions, depending on the expression dynamics. When Ascl1 expression oscillates, NPCs proliferate actively. By contrast, when the expression is sustained, NPCs differentiate into post-mitotic neurons. Further analyses will be required to understand the mechanism by which these bHLH factors lead to different outcomes depending on different expression dynamics.

bHLH factors seem to be useful tools for regenerative medicine of neural tissues. We have recently developed a new optogenetics method that enables oscillatory versus sustained gene expression. This method offers a new way to control the proliferation and differentiation of stem cells by simply changing the light exposure pattern rather than using different growth factors or chemicals, showing its applicability to the regeneration technology.

\section{References}

Akazawa C, Sasai Y, Nakanishi S, Kageyama R (1992) Molecular characterization of a 
rat negative regulator with a basic helix-loop-helix structure predominantly expressed in the developing nervous system. J Biol Chem 267:21879-21885.

Ali F, Hindley C, McDowell G, Deibler R, Jones A, Kirschner M, Guillemot F, Philpott A (2011) Cell cycle-regulated multi-site phosphorylation of Neurogenin 2 coordinates cell cycling with differentiation during neurogenesis. Development 138:4267-4277.

Alvarez-Buylla A, Garcia-Verdugo JM, Tramontin AD (2001) A unified hypothesis on the lineage of neural stem cells. Nat Rev Neurosci 2:287-293.

Baek JH, Hatakeyama J, Sakamoto S, Ohtsuka T, Kageyama R (2006) Persistent and high levels of Hes 1 expression regulate boundary formation in the developing central nervous system. Development 133:2467-2476.

Bertrand N, Castro DS, Guillemot F (2002) Proneural genes and the specification of neural cell types. Nat Rev Neurosci 3:517-530.

Castro DS, Skowronska-Krawczyk D, Armant O, Donaldson IJ, Parras C, Hunt C, Critchley JA, Nguyen L, Gossler A, Gottgens B, Mtter JM, Guillemot F (2006) Proneural bHLH and Brn proteins coregulate a neurogenic program through cooperative binding to a conserved DNA motif. Dev Cell 11:831-844.

Fietz SA, Kelava I, Vogt J, Wilsch-Bräuninger M, Stenzel D, Fish JL, Corbeil D, Riehn A, Distler W, Nitsch R, Huttner WB (2010) OSVZ progenitors of human and ferret neocortetx are epithelial-like and expand by integrin signaling. Nat Neurosci 13:690-699.

Fishell G, Kriegstein AR (2003) Neurons from radial glia: the consequences of asymmetric inheritance. Curr Opin Neurobiol 13:34-41.

Fortini ME (2009) Notch signaling: the core pathway and its posttranslational regulation. Dev Cell 16:633-647. 
Fujita S (2003) The discovery of the matrix cell, the identification of the multipotent neural stem cell and the development of the central nervous system. Cell Struct Funct 28:205-228.

Grbavec D, Stifani S (1996) Molecular interaction between TLE1 and the carboxyl-terminal domain of HES-1 containing the WRPW motif. Biochem Biophys Res Commun 223:701-705.

Götz M, Huttner WB (2005) The cell biology of neurogenesis. Nat Rev Mol Cell Biol 6:777-788.

Hansen DV, Lui JH, Parker PRL, Kriegstein AR (2010) Neurogenic radial glia in the outer subventricular zone of human neocortex. Nature 464:554-561.

Hatakeyama J, Bessho Y, Katoh K, Ookawara S, Fujioka M, Guillemot F, Kageyama R (2004) Hes genes regulate size, shape and histogenesis of the nervous system by control of the timing of neural stem cell differentiation. Development 131:5539-5550.

Hindley C, Ali F, McDowell G, Cheng K, Jones A, Guillemot F, Philpott A (2012) Post-translational modification of Ngn2 differentially affects transcription of distinct targets to regulate the balance between progenitor maintenance and differentiation. Development 139:1718-1723.

Hirabayashi Y, Suzki N, Tsuboi M, Endo TA, Toyoda T, Shinga J, Koseki H, Vidal M, Gotoh Y (2009) Polycomb limits the neurogenic competence of neural precursor cells to promote astrogenic fate transition. Neuron 63:600-613.

Hirata H, Tomita K, Bessho Y, Kageyama R (2001) Hes1 and Hes3 regulate maintenance of the isthmic organizer and development of the mid/hindbrain. EMBO J 20:4454-4466.

Hirata H, Yoshiura S, Ohtsuka T, Bessho Y, Harada T, Yoshikawa K, Kageyama R 
(2002) Oscillatory expression of the bHLH factor Hes 1 regulated by a negative feedback loop. Science 298:840-843.

Hirata H, Bessho Y, Kokubu H, Masamizu Y, Yamada S, Lewis J, Kageyama R (2004) Instability of Hes7 protein is critical for the somite segmentation clock. Nat Genet 36:750-754.

Honjo $\mathrm{T}$ (1996) The shortest path from the surface to the nucleus: RBP-J k/Su(H) transcription factor. Genes Cells 1:1-9.

Imayoshi I, Sakamoto M, Yamaguchi M, Mori K, Kageyama R (2010) Essential roles of Notch signaling in maintenance of neural stem cells in the developing and adult brains. J Neurosci 30:3489-3498.

Imayoshi I, Isomura A, Harima Y, Kawaguchi K, Kori H, Miyachi H, Fujiwara TK, Ishidate F, Kageyama R (2013) Oscillatory control of factors determining multipotency and fate in mouse neural progenitors. Science 342:1203-1208.

Ishibashi M, Moriyoshi K, Sasai Y, Shiota K, Nakanishi S, Kageyama R (1994) Persistent expression of helix-loop-helix factor HES-1 prevents mammalian neural differentiation in the central nervous system. EMBO J 13:1799-1805.

Ishibashi M, Ang S-L, Shiota K, Nakanishi S, Kageyama R, Guillemot F (1995) Targeted disruption of mammalian hairy and Enhancer of split homolog-1 (HES-1) leads to up-regulation of neural helix-loop-helix factors, premature neurogenesis and severe neural tube defects. Genes Dev 9:3136-3148.

Jarriault S, Brou C, Logeat F, Schroeter EH, Kopan R, Israel A. (1995) Signalling downstream of activated mammalian Notch. Nature 377:355-358.

Kageyama R, Ohtsuka T, Kobayashi T (2007) The Hes gene family: repressors and oscillators that orchestrate embryogenesis. Development 134:1243-1251. 
Kageyama R, Ohtsuka T, Shimojo H, Imayoshi I (2008) Dynamic Notch signaling in neural progenitor cells and a revised view of lateral inhibition. Nat Neurosci 11:1247-1251.

Kawaguchi A, Ikawa T, Kasukawa T, Ueda HR, Kurimoto K, Saitou M, Matsuzaki F (2008) Single-cell gene profiling defines differential progenitor subclasses in mammalian neurogenesis. Development 135:3113-3124.

Kiecker C, Lumsden A (2005) Compartments and their boundaries in vertebrate brain development. Nat Rev Neurosci 6:553-564.

Knuckles P, Vogt MA, Lugert S, Milo M, Chong MM, Hautbergue GM, Wilson SA, Littman DR, Taylor V (2012) Drosha regulates neurogenesis by controlling neurogenin 2 expression independent of microRNAs. Nat Neurosci 15:962-969.

Kopan R, Ilagan MXG (2009) The canonical Notch signaling pathway: unfolding the activation mechanism. Cell 137:216-233.

Kriegstein A, Alvarez-Buylla A (2009) The glial nature of embryonic and adult neural stem cells. Annu Rev Neurosci 32:149-184.

Malatesta P, Hartfuss E, Götz M (2000) Isolation of radial glial cells by fluorescent-activated cell sorting reveals a neuronal lineage. Development 127:5253-5263.

Masamizu Y, Ohtsuka T, Takashima Y, Nagahara H, Takenaka Y, Yoshikawa K, Okamura H, Kageyama R (2006) Real-time imaging of the somite segmentation clock: revelation of unstable oscillators in the individual presomitic mesoderm cells. Proc Natl Acad Sci USA 103:1313-1318.

Miller FD, Gauthier AS (2007) Timing is everything: making neurons versus glia in the developing cortex. Neuron 54:357-369. 
Miyata T, Kawaguchi A, Okano H, Ogawa M (2001) Asymmetric inheritance of radial glial fibers by cortical neurons. Neuron 31:727-741.

Mizutani K, Yoon K, Dang L, Tokunaga A, Gaiano N (2007) Differential Notch signalling distinguishes neural stem cells from intermediate progenitors. Nature 449:351-355.

Noctor SC, Flint AC, Weissman TA, Dammerman RS, Kriegstein AR (2001) Neurons derived from radial glial cells establish radial units in neocorttex. Nature 409:714-720.

Ohtsuka T, Ishibashi M, Gradwohl G, Nakanishi S, Guillemot F, Kageyama R (1999) Hes 1 and Hes5 as Notch effectors in mammalian neuronal differentiation. EMBO J 18:2196-2207.

Ohtsuka T, Sakamoto M, Guillemot F, Kageyama R (2001) Roles of the Basic Helix-Loop-Helix Genes Hes1 and Hes5 in Expansion of Neural Stem Cells of the Developing Brain. J Biol Chem 276:30467-30474.

Pierfelice T, Alberi L, Gaiano N (2011) Notch in the vertebrate nervous system: an old dog with new tricks. Neuron 69:840-855.

Ross SE, Greenberg ME, Stiles CD (2003) Basic helix-loop-helix factors in cortical development. Neuron 39:13-25.

Sang L, Coller HA, Roberts JM (2008) Control of the reversibility of cellular quiescence by the transcriptional repressor HES1. Science 321:1095-1100.

Sasai Y, Kageyama R, Tagawa Y, Shigemoto R, Nakanishi S (1992) Two mammalian helix-loop-helix factors structurally related to Drosophila hairy and Enhancer of split. Genes Dev 6:2620-2634.

Sessa A, Mao C, Hadjantonakis AK, Klein WH, Broccoli V (2008) Tbr2 directs conversion of radial glia into basal precursors and guides neuronal amplification by 
indirect neurogenesis in the developing neocortex. Neuron 60:56-69.

Shimojo H, Ohtsuka T, Kageyama R (2008) Oscillations in Notch signaling regulate maintenance of neural progenitors. Neuron 58:52-64.

Shitamukai A, Konno D, Matsuzaki F (2011) Oblique radial glial divisions in the developing mouse neocortex induce self-renewing progenitors outside the germinal zone that resemble primate outer subventricular zone progenitors. J Neurosci 31:3683-3695.

Sriuranpong V, Borges MW, Strock CL, Nakakura EK, Watkins DN, Blaumueller CM, Nelkin BD, Ball DW (2002) Notch signaling induces rapid degradation of achaete-scute homolog 1. Mol Cell Biol 22:3129-3139.

Takebayashi K, Sasai Y, Sakai Y, Watanabe T, Nakanishi S, Kageyama R (1994) Structure, chromosomal locus, and promoter analysis of the gene encoding the mouse helix-loop-helix factor HES-1: negative autoregulation through the multiple $\mathrm{N}$ box elements. J Biol Chem 269:5150-5156.

Tan S-L, Nishi M, Ohtsuka T, Matsui T, Takemoto K, Kamio-Miura A, Aburatani H, Shinkai Y, Kageyama R (2012) Essential roles of the histone methyltransferase ESET in the epigenetic control of neural progenitor cells during development. Development 139:3806-3816.

Wang X, Chen X, Yang Y (2012) Spatiotemporal control of gene expression by a light-switchable transgene system. Nature Meth 9:266-269.

Wilkinson G, Dennis D, Schuurmans C (2013) Proneural genes in neocortical development. Neuroscience 253:256-273.

Yoshiura S, Ohtsuka T, Takenaka Y, Nagahara H, Yoshikawa K, Kageyama R (2007) Ultradian oscillations of Stat, Smad, and Hes1 expression in response to serum. Proc Natl Acad Sci USA 104:11292-11297. 


\section{Figure legends}

Figure 1. Neural progenitor cells and their differentiation in the embryonic brain. Neuroepithelial cells initially undergo self-renewal by symmetric division and proliferate extensively. As development proceeds, neuroepithelial cells are elongated to become radial glial cells, which have cell bodies in the inner region (the ventricular zone) of the neural tube and radial fibers that reach the pial surface. Radial glial cells give rise to neurons first. After the production of neurons, radial glial cells give rise to oligodendrocytes, ependymal cells, and astrocytes. Both neuroepithelial cells and radial glial cells are considered embryonic neural progenitor cells.

Figure 2. The core pathway of Notch signaling.

Proneural factors such as Ascl1 and Neurog2, which promote neuronal differentiation, induce the expression of Notch ligands such as Dll1 (Cell 1), which activate Notch signaling in neighboring cells (Cell 2). Upon activation, Notch is cleaved at the transmembrane part, releasing the Notch intracellular domain (NICD). NICD then moves to the nucleus, where it forms a complex with the DNA-binding protein Rbpj and the transcriptional co-activator Maml. The NICD-Rbpj-Maml ternary complex induces the expression of Hes1 and Hes5, which repress the expression of proneural genes and Dll1, thereby leading to the maintenance of neural progenitor cells (Cell 2).

Figure 3. Oscillatory expression of Hes 1 by negative feedback.

Hes 1 expression oscillates by negative feedback with a period of 2-3 hours in neural progenitor cells. Hes 1 represses its own expression by directly binding to its promoter. This negative feedback leads to the disappearance of hes 1 mRNA and Hes1 protein, because they are extremely unstable, allowing the next round of its expression. In this way, Hes1 autonomously starts oscillatory expression.

Figure 4. Expression dynamics in neural progenitor cells and differentiating neurons.

Hes 1 expression oscillates with a period of 2-3 hours in neural progenitor cells. In these cells, Hes1 oscillation drives the oscillatory expression of Ascl1, Neurog2, and Dll1 by 
periodic repression. When Hes1 expression disappears, Ascl1, Neurog2, and Dll1 expression becomes sustained, promoting neuronal differentiation. Thus, the oscillatory expression correlates with proliferation of neural progenitor cells, whereas sustained expression correlates with neuronal differentiation.

Figure 5. Basal progenitors and OSVZ progenitors.

Basal progenitors retract apical and basal processes and generally divide only once to generate two neurons in the subventricular zone. In these cells, Hesl expression is down-regulated, suggesting that Notch signaling is not active. OSVZ progenitors have radial glia-like morphology extending radial fibers to the pial surface but lack apical processes. These cells repeatedly undergo asymmetric cell division, each dividing into a daughter cell that inherits the radial fiber (OSVZ progenitor) and a neuron that does not. Neurons express Notch ligands and activate Notch signaling in their sibling OSVZ progenitors.

Figure 6. Different expression dynamics of Hes1 in the developing nervous system.

The developing nervous system is partitioned into many compartments by boundaries such as the isthmus and the zona limitans intrathalamica (Zli). The nervous system is also partitioned into the right and left halves by the roof plate and the floor plate. Cells in boundary regions are mostly dormant with regard to proliferation and differentiation, in contrast to neural progenitor cells present in compartments. Boundary cells express Hes 1 in a sustained manner while neural progenitor cells present in compartments express Hes1 in an oscillatory manner.

Figure 7. Hypothesis for the involvement of Hes 1 and proneural factor oscillators in the temporal fate switch of NPCs. ESET activity gradually decreases while Polycomb group complex (PcG) activity on proneural genes may increase over time, and these factors regulate the temporal fate switch of NPCs. Their expression might be directly or indirectly controlled by Hes1 and proneural factor oscillators, although the exact mechanism is unknown. 
Figure 1

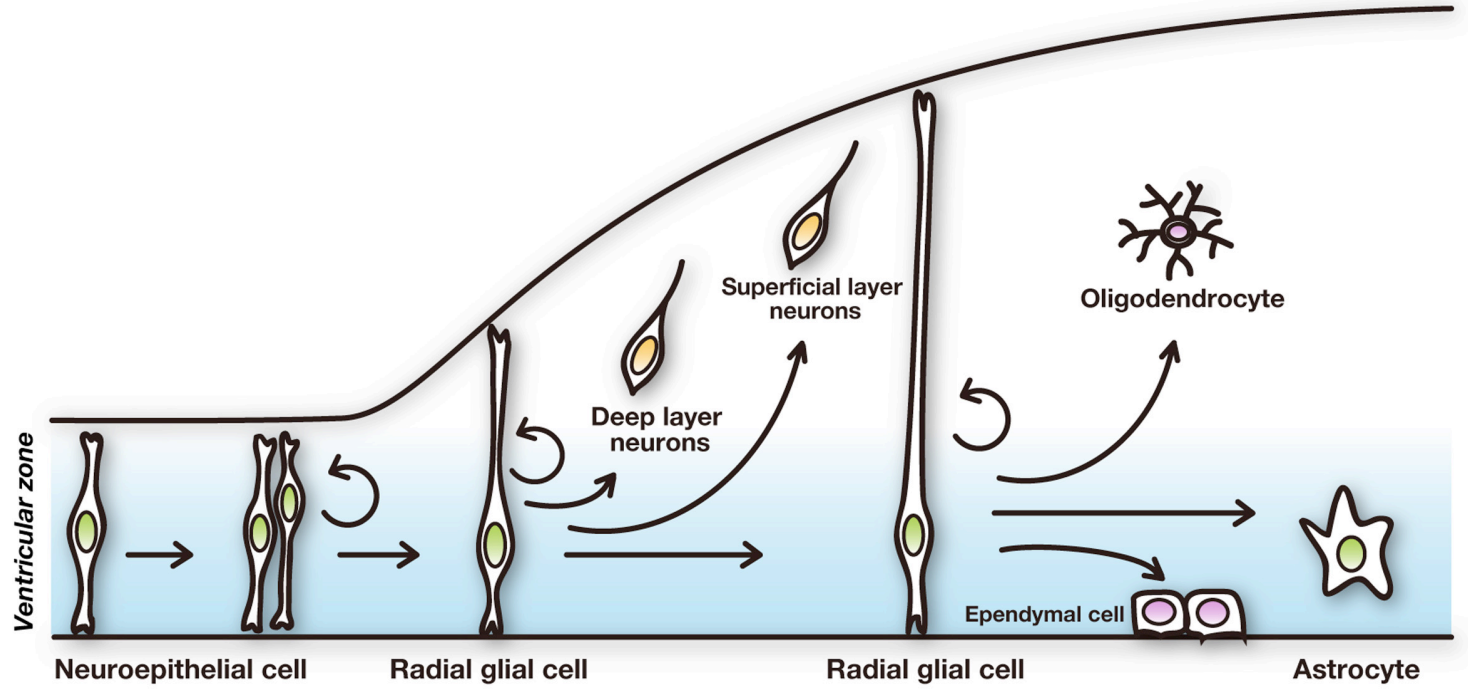

Figure 2

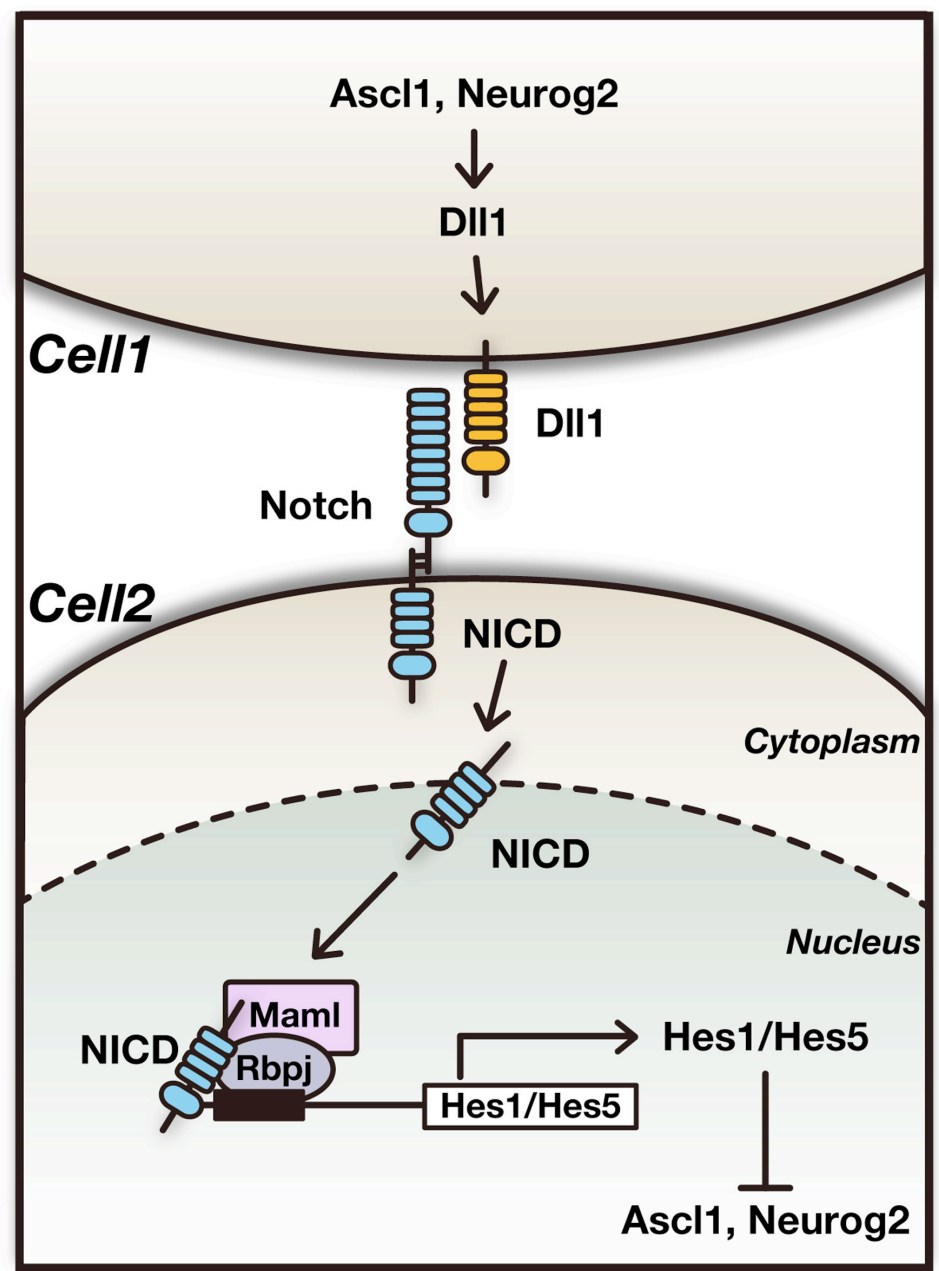


Figure 3

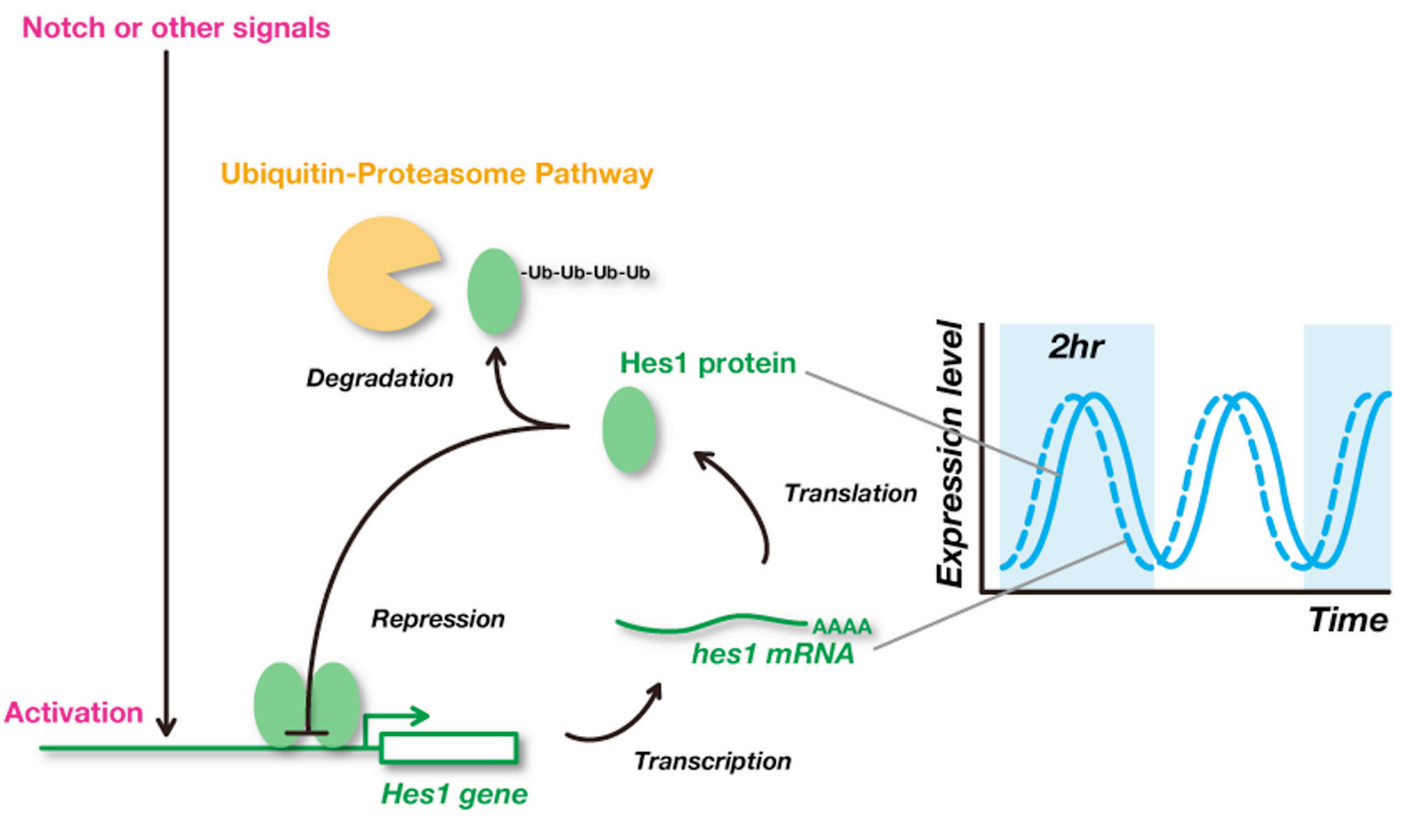

Figure 4

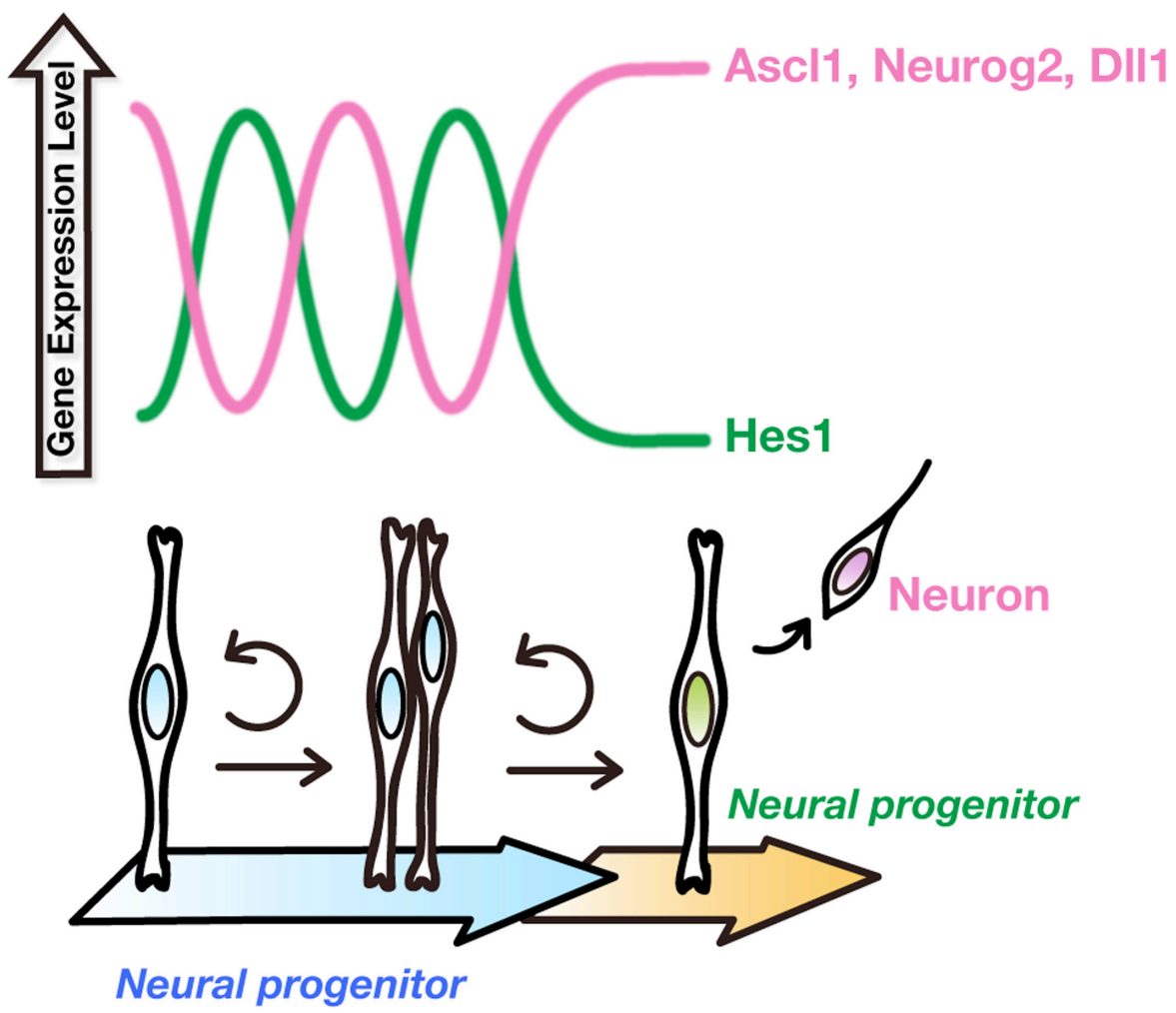


Figure 5

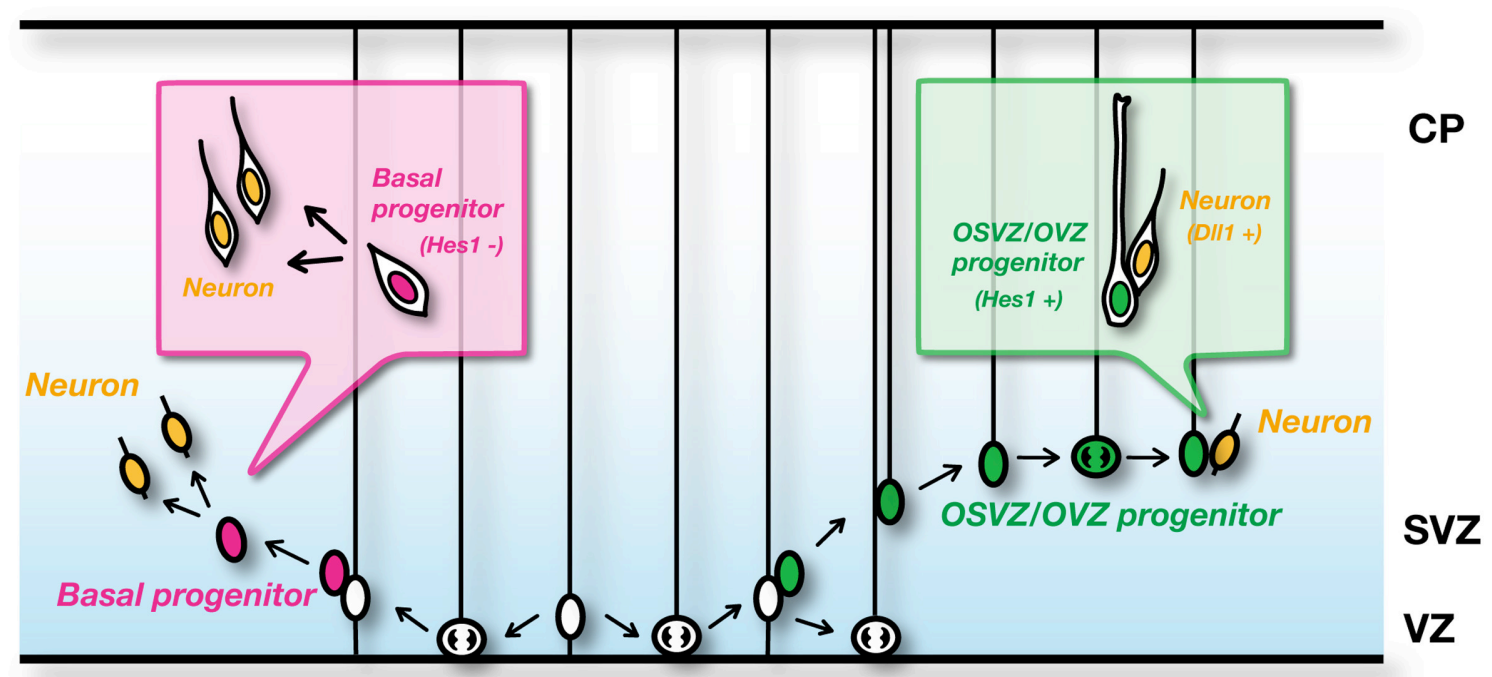

Figure 6

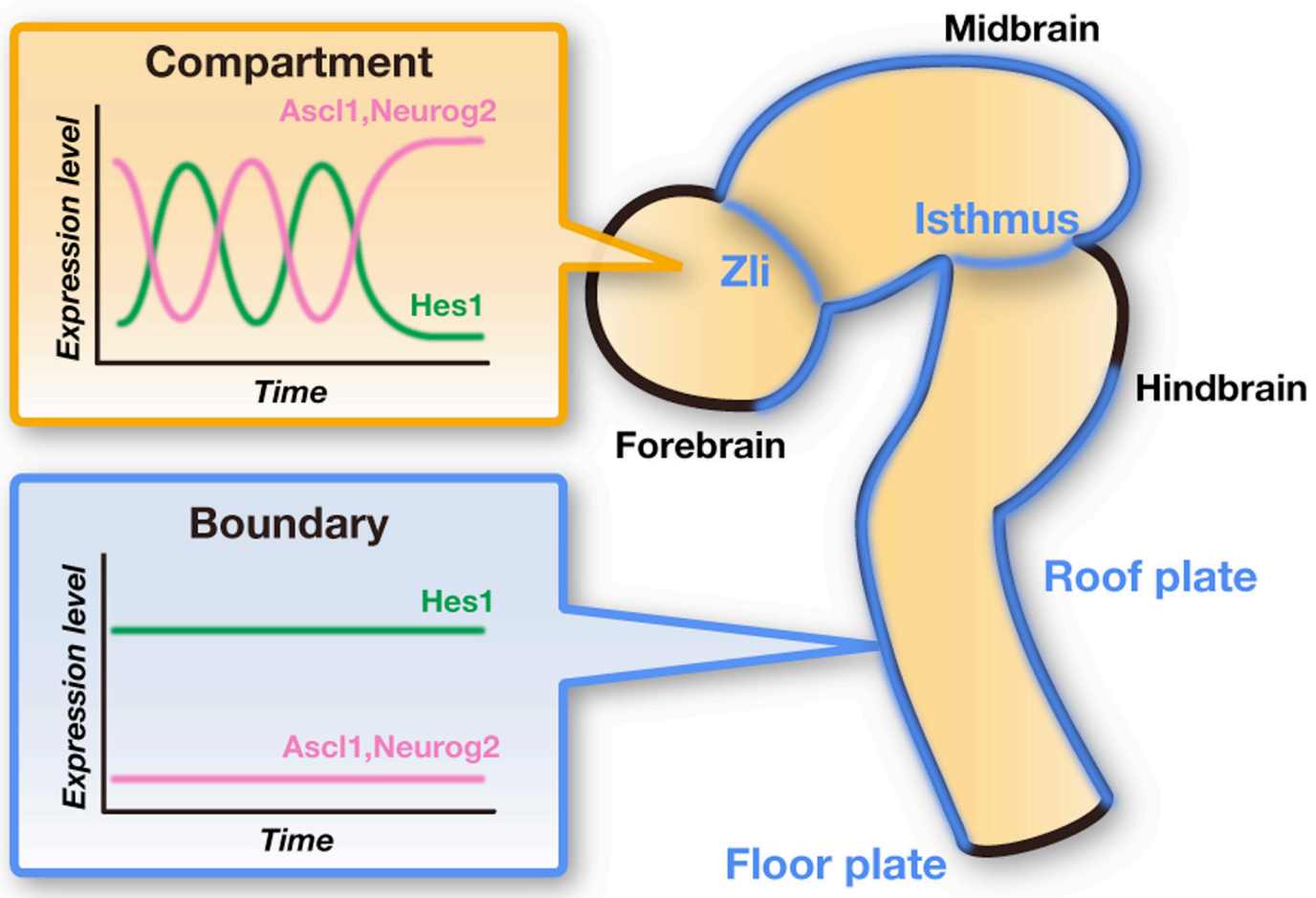


Figure 7
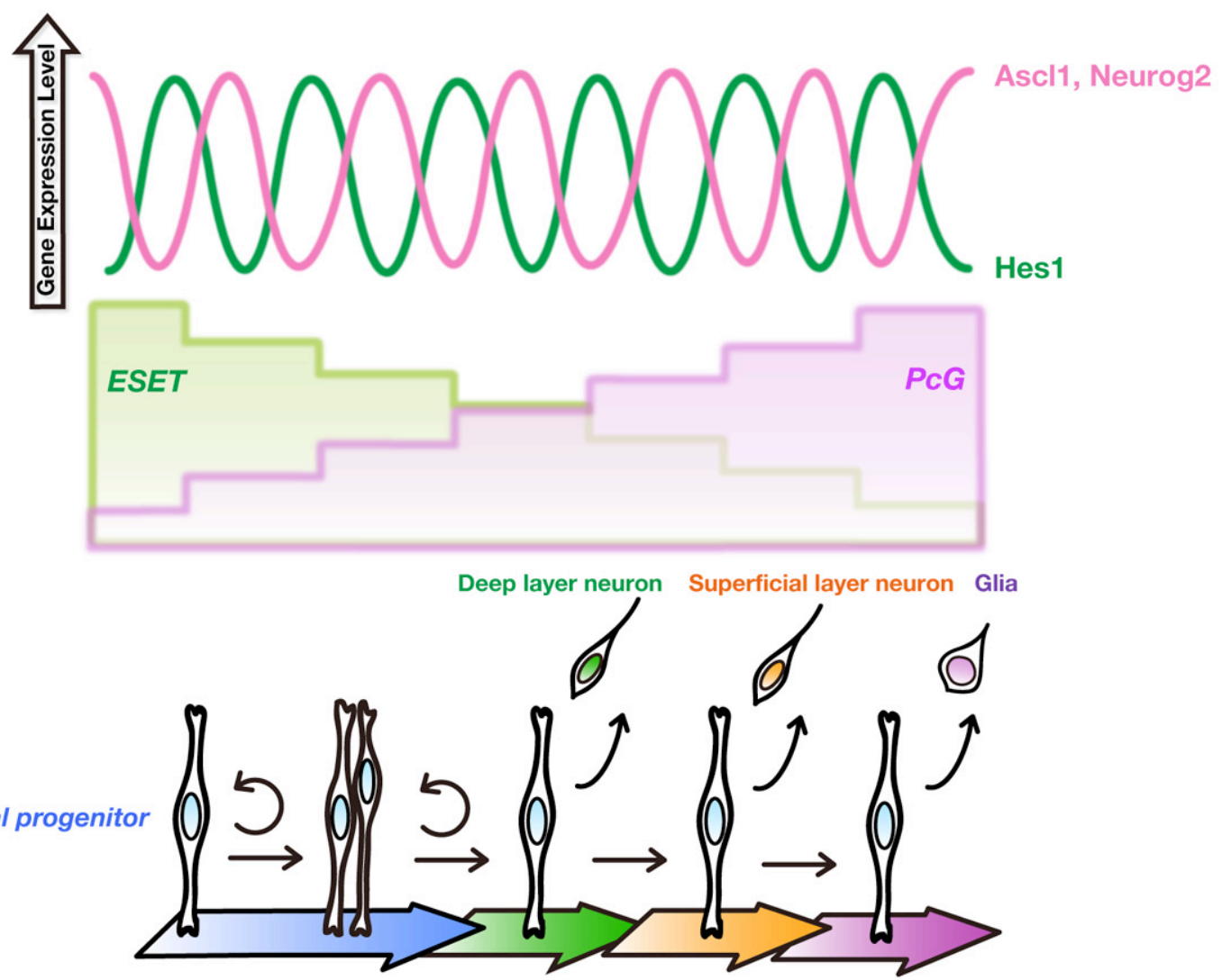